\title{
Statistical Analysis of Size Effects on Time-Dependent Fracture of an Alumina
}

\author{
Chih-Kuang LIN* and Wen-Shao CHEN \\ Department of Mechanical Engineering, National Central University, Chung-Li 32054, Taiwan, R. O. C.
}

\author{
アルミナの時間依存型破壊の寸法効果の統計的解析 \\ 林 志光・陳 文紹 \\ 國立中央大學工學部機械工學科，32054 中華民國台灣省中壢市
}

\begin{abstract}
Time-dependent fracture of an alumina under long-term static and cyclic loading was investigated by fourand three-point flexure tests. Bend bars with two different sizes were prepared to evaluate the size effects on the failure time distribution. The resulting stress-life data were analyzed by two statistical time-dependent fracture theories which incorporate the statistics of strength and flaw size distribution with subcritical crack growth that leads to delayed fracture. The first model considers only the growth of a single major crack to a critical size while the second considers the size distribution of multiple flaws and their slow crack growth characteristics. Both models provided commonly acceptable description of the static and cyclic strength-probability-lifetime relationships for the experimental results from various specimen sizes and flexure modes.

[Received September 9, 1996; Accepted June 6, 1997]
\end{abstract}

Key-words : Statistical analysis, Time-dependent fracture, Size effect, Alumina, Subcritical crack growth

\section{Introduction}

Strength values of ceramics obtained from nominally identical specimens tested under similar conditions are usually dispersed, as the fracture-initiating flaws vary from specimen to specimen. Therefore, the strength of ceramics is not unique for a given size and also dependent on component size. A number of studies ${ }^{1)-3}$ on ceramics have examined the relationships between strengths in different specimen sizes. Large specimens have lower failure stresses than small ones simply because a more severe flaw is likely to exist among the greater number of flaws in the large specimen. Such sensitivity to size effect of strength suggests that probabilistic design concepts be more appropriate for ceramic components.

Since ceramics can exhibit a delayed fracture behavior under time-dependent loads, engineering design for ceramics should also take into account the phenomenon of delayed fracture. The scatter in strength of ceramics suggests that lifetime of ceramic components under time-dependent loads will also show considerable dispersion. Here, failure probabilities increase with service and have to be estimated as a function of service time. Hence, engineering design for ceramics requires an approach that deals with the probability of failure under a given applied stress. In this regard, studies (e.g., Refs. 4-12) were initiated to develop techniques for lifetime prediction using fracture mechanics data in conjunction with statistical analysis. However, there are very few experimental data to study the effects of specimen size on the delayed fracture of ceramics subjected to long-term mechanical loading with the applications of these proposed models. The increasing application of ceramics as structural components increases the importance of such study to interpret the size effects on delayed fracture and to ensure the reliability of components.

In the present study, a complete set of self-contained data obtained under static and cyclic loading for a commercial alumina are analyzed in accordance with two statistical fracture models. The size effects on the fatigue lifetime distribution under long-term mechanical loading are examined between two specimen sizes in four- and three-point flexure tests. It is the purpose of this paper to describe how the size effects on statistical variations in strength and lifetime distribution under a particular time-dependent stress state can be expressed by the strength-probability-lifetime relationship using information derived from simple experimental techniques.

\section{Statistical fracture theories}

For reasons of simplicity only uniform specimens with rectangular cross-sections subjected to three- and fourpoint flexure are considered in the statistical fracture approaches introduced in this section. Similar results can also be obtained for other types of loading conditions through similar derivation procedures.

\subsection{Statistics of strength}

The most accepted statistical theory used in the analysis of fracture strength of brittle material is Weibull's weakest link theory. ${ }^{13)}$ For volume distributed flaws the failure probability $F$ at a maximum applied stress $\sigma_{\mathrm{m}}$ is given by ${ }^{14)}$

$$
F\left(\sigma_{\mathrm{m}}\right)=1-\exp \left[-\int_{V} \int_{a\left(\sigma_{x y}\right)}^{\infty} q(a) \mathrm{d} a \mathrm{~d} V\right]
$$

where $\sigma_{x y}$ is the maximum principal stress at position $(x, y)$ within the stressed volume when $\sigma_{\mathrm{m}}$ is applied, $q(a)$ is a flaw size distribution function, $a$ is the equivalent length of the Griffith flaw lying normal to the maximum principal stress, and $V$ is the stressed volume of the material. According to Griffith's fracture theory,

$$
a\left(\sigma_{x y}\right)=\left[\frac{K_{\mathrm{IC}}}{Y \sigma_{x y}}\right]^{2}
$$

where $K_{\mathrm{IC}}$ is the fracture toughness and $Y$ is a geometric factor. Assuming a Pareto distribution function for $q(a)$ such that ${ }^{8), 14)}$

$$
q(a)= \begin{cases}\frac{m \rho_{\mathrm{f}}}{2 a_{0}}\left(\frac{a_{0}}{a}\right)^{m+2 / 2}, & a \geq a_{0} \\ 0, & a<a_{0}\end{cases}
$$

where $a_{0}$ is the smallest flaw size, $\rho_{\mathrm{f}}$ is the flaw density, and $m$ is a constant. No interaction between flaws is considered.

Integrating Eq. (1) over the volume $V$ of the stressed 
material between the two outer support points in flexure test yields the most frequently observed two-parameter Weibull function ${ }^{1)}$

$$
F\left(\sigma_{\mathrm{m}}\right)=1-\exp \left[-\frac{(m+2) V}{4(m+1)^{2}}\left(\frac{\sigma_{\mathrm{m}}}{\sigma_{0}}\right)^{m}\right]
$$

for four-point flexure, and

$$
F\left(\sigma_{\mathrm{m}}\right)=1-\exp \left[-\frac{V}{2(m+1)^{2}}\left(\frac{\sigma_{\mathrm{m}}}{\sigma_{0}}\right)^{m}\right]
$$

for three-point flexure, where a scale parameter $\sigma_{0}$ is defined by

$$
\sigma_{0}=\left[\frac{K_{\mathrm{IC}}{ }^{m}}{\rho_{\mathrm{f}} a_{0}{ }^{m / 2} Y^{m}}\right]^{1 / m}
$$

here, $m$ is the Weibull modulus and $V$ is the volume between the two outer support points in a bend bar. Similar scaling relationships (but with stressed surface areas on the right-hand sides of Eqs. (4) and (5)) can be obtained if surface flaws are strength limiting. However, Eqs. (4) and (5) for volume flaws would be used throughout this study based on a more general consideration, as experimental results indicated that some of the fracture origins in the present work were associated with subsurface or volume defects.

\subsection{Statistics of time-dependent fracture}

On the basis of the observation of delayed failure and subcritical crack growth in many ceramic materials, it is common and appropriate to assume that delayed failure results from the growth of small cracks or other crack-like flaws. Consequently, crack propagation is usually the prevailing failure process in ceramics. Lifetime prediction for ceramics is thus possible by applying the crack propagation analysis. This will be a conservative underestimation if crack initiation becomes significant in failure process.

To study delayed fracture of ceramics, all pre-existing flaws are assumed to propagate in accordance with a subcritical crack growth law. ${ }^{15)}$ Two well-established approaches based on this subcritical crack growth law are introduced to analyze the time-dependent fracture strength data generated in the current work. The first model is based on the assumption that the flaws controlling strength are usually identical for both instantaneous fracture and delayed fracture. ${ }^{6)}$ However, the second model takes into account the growth of all pre-existing flaws under nonuniform stresses and how this growth influences the original flaw size distribution and the associated lifetime in delayed failure. ${ }^{8)}$

\subsubsection{Single-crack model}

In this model only the growth of a single crack from an initial flaw size to a critical size is considered. Subcritical crack growth in ceramics is usually described by the following power law: ${ }^{15)}$

$$
\nu=\frac{\mathrm{d} a}{\mathrm{~d} t}=A K_{\mathrm{I}}^{n}=A(Y \sigma \sqrt{a})^{n}
$$

where $v$ is the crack velocity during crack propagation, $a$ is the crack size, $t$ is time, $A$ and $n$ are material constants, $K_{\mathrm{I}}$ is the stress intensity factor of mode I, $Y$ is a geometric factor and $\sigma$ is the applied stress.

The applied stress, $\sigma$, which is a function of time in delayed fracture, can be generally expressed as

$$
\sigma=\sigma_{\mathrm{m}} f(t)
$$

where $\sigma_{\mathrm{m}}$ is the maximum applied stress during the entire loading period and $0 \leq f(t) \leq 1$, as only tensile stresses are considered to contribute to crack growth. For static fatigue tests with sustained constant loading, where $f(t)=1$, the static lifetime, $t_{\mathrm{sf}}$, can be obtained by integration of Eq. (6) such that ${ }^{16)}$

$$
t_{\mathrm{sf}} \sigma_{\mathrm{m}}^{n}=\frac{2}{(n-2) A Y^{n}} a_{\mathrm{i}}^{2-n / 2}
$$

where $a_{\mathrm{i}}$ is the initial flaw size. For fatigue tests performed under a cyclic loading wave form with a period $\tau$, the cyclic lifetime, $t_{\mathrm{cf}}$, can also be obtained as follows:16)

$$
t_{\mathrm{cf}} \sigma_{\mathrm{m}}^{n}=\frac{2}{(n-2) A Y^{n}} a_{\mathrm{i}}^{2-n / 2} \tau\left\{\int_{0}^{\tau}[f(t)]^{n} \mathrm{~d} t\right\}^{-1}
$$

The power laws expressed in Eqs. (8) and (9) between applied stress and corresponding lifetime are often applied in conventional analysis of delayed fracture of ceramics without statistical consideration.

The initial flaw size $a_{\mathrm{i}}$ is related to the instantaneous fracture strength $S$ by the Griffith theory, $S Y \sqrt{a_{i}}=K_{\text {IC }}$. With such relationship and Eqs. (4), (8) and (9), the statistical effects are now combined with the above time-dependent failure analysis to give the strength-probability-lifetime relationship. Accordingly, the strength-probability-lifetime relationships for ceramics in four-point flexure can be expressed as ${ }^{6), 17)}$

$$
\begin{aligned}
t_{\mathrm{st}} \sigma_{\mathrm{m}}^{n}= & \frac{2}{(n-2) A Y^{2} K_{\mathrm{IC}}^{n-2}}\left[\frac{4(m+1)^{2} \sigma_{0}^{m}}{(m+2) V}\right. \\
& \left.\times \ln \frac{1}{1-F}\right]^{n-2 / m}
\end{aligned}
$$

for static loading with a constant stress $\sigma_{\mathrm{m}}$, and

$$
\begin{aligned}
t_{\mathrm{cf}} \sigma_{\mathrm{m}}^{n}= & \frac{2}{(n-2) A Y^{2} K_{\mathrm{IC}}^{n-2}}\left[\frac{4(m+1)^{2} \sigma_{0}^{m}}{(m+2) V}\right. \\
& \left.\times \ln \frac{1}{1-F}\right]^{n-2 / m} \tau\left\{\int_{0}^{\tau}[f(t)]^{n} \mathrm{~d} t\right\}^{-1}
\end{aligned}
$$

for cyclic loading with a peak stress $\sigma_{\mathrm{m}}$. In Eqs. (10) and (11), the probability of failure at a certain time under a given loading condition is related to the probability of finding a flaw bigger than $a_{\mathrm{i}}$ on the assumption that the flaw causing failure in rapid loading also causes delayed fracture under time-dependent loading. Similar results for threepoint flexure can be obtained as

$$
\begin{aligned}
t_{\mathrm{sf}} \sigma_{\mathrm{m}}^{n}= & \frac{2}{(n-2) A Y^{2} K_{\mathrm{IC}}^{n-2}}\left[\frac{2(m+1)^{2} \sigma_{0}^{m}}{V}\right. \\
& \left.\times \ln \frac{1}{1-F}\right]^{n-2 / m}
\end{aligned}
$$

for static fatigue loading, and

$$
\begin{aligned}
t_{\mathrm{cf}} \sigma_{\mathrm{m}}^{n}= & \frac{2}{(n-2) A Y^{2} K_{\mathrm{IC}}^{n-2}}\left[\frac{2(m+1)^{2} \sigma_{0}^{m}}{V}\right. \\
& \left.\times \ln \frac{1}{1-F}\right]^{n-2 / m}
\end{aligned}
$$

for cyclic fatigue loading.

\subsubsection{Multiple-crack model}

The sizes of pre-existing flaws in ceramics are not constant and they may be randomly distributed within the volume. Therefore, another statistical fracture approach was initiated by $\mathrm{Hu}$ et al. ${ }^{8)}$ to include flaw statistics in predicting time-dependent strength behavior, in particular for otherwise smooth specimens subjected to nonuniform stresses. The change of flaw size distribution due to subcritical crack growth of all cracks within stressed volume during time-dependent loading is taken into account in this multiple-crack model.

For a flaw located at $(x, y)$ and subjected to a given stress $\sigma_{x y}$, its growth from a reference size $a_{\mathrm{r}}$ at $t=0$ to size $a$ at time $t$ is obtained from Eq. (6), where 


$$
\int_{a_{\mathrm{r}}}^{a} a^{-n / 2} \mathrm{~d} a=\int_{0}^{t} A\left(\sigma_{x y} Y\right)^{n} \mathrm{~d} t
$$

The flaw size distribution function, thus, changes from $q\left(a_{\mathrm{r}}\right.$, $0)\left[=q\left(a_{\mathrm{r}}\right)\right]$ to $q(a, t)$ due to the growth of flaws within the stressed volume $V$. The failure probability equation at time $t$ can be determined as ${ }^{8)}$

$$
F\left(\sigma_{\mathrm{m}}, t\right)=1-\exp \left[-\int_{V} \int_{a_{\mathrm{r}}\left(\sigma_{x y}, t\right)}^{\infty} q(a) \mathrm{d} a \mathrm{~d} V\right]
$$

The strength-probability-lifetime equations for delayed fracture can be obtained from Eqs. (3), (14) and (15) with the consideration that $\sigma_{x y}$ varies generally across beam depth and along beam length in a rectangular bend bar under loading. For the case of delayed fracture under a sustained constant stress $\sigma_{\mathrm{m}}$ in four-point flexure, the following results can be obtained for volume-distributed flaws, ${ }^{8), 17)}$

$$
\begin{aligned}
t_{\mathrm{sf}} \sigma_{\mathrm{m}}^{n}= & \frac{2}{(n-2) A Y^{2} K_{\mathrm{IC}}^{n-2}}\left[\frac{4\left(m_{*}+1\right)^{2} \sigma_{0}^{m}}{V\left(m_{*}+2\right)}\right. \\
& \left.\times \ln \frac{1}{1-F}\right]^{n-2 / m}
\end{aligned}
$$

where parameter $m_{*}$ is defined as $m_{*}=m n / n-2$. For fourpoint flexure with cyclic loading, where $\sigma_{\mathrm{m}}$ is the peak stress, the strength-probability-lifetime equation becomes $^{8), 17)}$

$$
\begin{aligned}
t_{\mathrm{cf}} \sigma_{\mathrm{m}}^{n}= & \frac{2}{(n-2) A Y^{2} K_{\mathrm{IC}}^{n-2}}\left[\frac{4\left(m_{*}+1\right)^{2} \sigma_{0}^{m}}{V\left(m_{*}+2\right)}\right. \\
& \left.\times \ln \frac{1}{1-F}\right]^{n-2 / m} \tau\left\{\int_{0}^{\tau}[f(t)]^{n} \mathrm{~d} t\right\}^{-1}
\end{aligned}
$$

Similar results for three-point flexure in static and cyclic fatigue loading are given by

$$
\begin{aligned}
t_{\mathrm{sf}} \sigma_{\mathrm{m}}^{n}= & \frac{2}{(n-2) A Y^{2} K_{\mathrm{IC}}^{n-2}}\left[\frac{2\left(m_{*}+1\right)^{2} \sigma_{0}^{m}}{V}\right. \\
& \left.\times \ln \frac{1}{1-F}\right]^{n-2 / m}
\end{aligned}
$$

and

$$
\begin{aligned}
t_{\mathrm{cf}} \sigma_{\mathrm{m}}^{n}= & \frac{2}{(n-2) A Y^{2} K_{\mathrm{IC}}^{n-2}}\left[\frac{2\left(m_{*}+1\right)^{2} \sigma_{0}^{m}}{V}\right. \\
& \left.\times \ln \frac{1}{1-F}\right]^{n-2 / m} \tau\left\{\int_{0}^{\tau}[f(t)]^{n} \mathrm{~d} t\right\}^{-1}
\end{aligned}
$$

respectively.

It is noted that the stressed volume $V$ in Eqs. (16) and (17) is slightly different from that in the original work of $\mathrm{Hu}$ et al. ${ }^{8}$ in which $V$ is the volume between the two inner loading points in four-point flexure. However, $V$ in the present study is the volume between the two outer support points for a more general consideration because the volume between the inner and outer points is also under stress. The formats of Eqs. (16) - (19) are similar to those of the counterparts in Eqs. (10)-(13) except for the difference between the constants $m$ and $m_{*}$ in the bracket terms on the right-hand sides. However, in physical meaning the lifetime predictions obtained in Eqs. (16) - (19) are different from those obtained in Eqs. (10)-(13). The single-crack model assumes that the origin of fracture is the same for both instantaneous and delayed failure. However, the multiplecrack model argues that in flexure and other nonuniformly stressed specimens, the failure causing flaw in instantaneous fracture is not necessarily the same as the flaw that leads to time-dependent fracture. The justification of these two approaches and their applicability to lifetime prediction for various time-dependent loading conditions will be eval- uated by the following experimental work.

\section{Experimental procedure}

In this study, experiments are designed to verify the lifetime predictions provided by the preceding analyses using the simple flexure test techniques with an emphasis on the size effects. The ceramic material selected for the present work is a commercial alumina (Aucera Technology Co., Taoyuan, Taiwan). The average grain size is about $12 \mu \mathrm{m}$ for this alumina with a density of $3.90 \mathrm{~g} / \mathrm{cm}^{3}$. The major impurity in this nominally $99.5 \%$ pure alumina is $\mathrm{MgO}$. Bendbar specimens with dimensions of two sizes, $3 \times 4 \times 45 \mathrm{~mm}$ (small) and $9 \times 12 \times 45 \mathrm{~mm}$ (large), were diamondmachined from sintered rectangular bars. The surfaces of each specimen were ground along its length with 300-grit diamond wheels. The four long edges of each specimen were chamfered to avoid premature fracture due to stress concentration. The dimensions of the small specimen and loading fixture were made as per ASTM Standard C1161. The large specimens were made for direct comparison to study the size effects on fatigue lifetime distribution. Both threeand four-point flexure tests were conducted with a support span of $40 \mathrm{~mm}$ and a load span of $20 \mathrm{~mm}$. These room-temperature tests were carried out in air using a closed-loop servohydraulic testing machine. Small specimens were used in the strength, static fatigue, and cyclic fatigue tests under four-point flexure to generate the base-line data for complete lifetime analysis and prediction. Static and cyclic fatigue tests under three- and four-point flexure were applied to the large specimens for evaluating the size effects on failure time distribution.

Fifteen small specimens were used to determine the flexural strength under four-point bending and the associated Weibull distribution and parameters. Fast fracture strength tests were performed under load control with a loading rate approximately $100 \mathrm{MPa} / \mathrm{s}$. Two types of delayed fracture tests have been adopted for both small and large specimens: static and cyclic fatigues. For static fatigue testing, specimens were subjected to several sustained constant loads until failure or runout. The cyclic fatigue tests were conducted at a stress ratio $R=\sigma_{\min } / \sigma_{\max }=0.1$ with a sinusoidal waveform and a frequency of $10 \mathrm{~Hz}$. Four peak stress levels, $170,190,210$ and $230 \mathrm{MPa}$, were applied on small specimens under four-point flexure in both static and cyclic tests. For the large-specimen category, both static and cyclic fatigue tests with three- and four-point flexure were conducted at four smaller peak stress levels, 110, 130, 150 and 170 $\mathrm{MPa}$ to generate comparable lifetime ranges with those obtained in small specimens. In both categories, five to ten

\begin{tabular}{|c|c|c|c|c|c|c|}
\hline \multirow{4}{*}{$\begin{array}{l}\text { Peak } \\
\text { stress } \\
(\mathrm{MPa})\end{array}$} & \multicolumn{6}{|c|}{ Number of specimens } \\
\hline & \multirow{2}{*}{\multicolumn{2}{|c|}{$\begin{array}{c}\text { Small size } \\
\text { Four-point }\end{array}$}} & \multicolumn{4}{|c|}{ Large size } \\
\hline & & & Fou & & Thre & oint \\
\hline & Static & Cyclic & Static & Cyclic & Static & Cyclic \\
\hline 230 & 10 & 5 & - & - & - & - \\
\hline 210 & 10 & 5 & - & - & - & - \\
\hline 190 & 10 & 5 & - & - & - & - \\
\hline 170 & 5 & 5 & 8 & 5 & 5 & 5 \\
\hline 150 & - & - & 8 & 5 & 5 & 5 \\
\hline 130 & - & - & 8 & 5 & 5 & 5 \\
\hline 110 & - & - & 5 & 5 & - & - \\
\hline
\end{tabular}
repeated tests were performed at each selected static and cyclic testing condition to obtain the lifetime distribution. Any specimen in both static and cyclic tests enduring $10^{5} \mathrm{~s}$ was considered to be a runout. Table 1 summarizes the testing program and the number of specimens used in each testing condition. Scanning electron microscopy (SEM) was conducted to identify the fracture origins of broken specimens in both fast- and delayed-fracture testings.

Table 1. Summary of Static and Cyclic Flexure Test Conditions* 


\section{Results and discussion,}

\subsection{Estimating values of $m$ and $n$}

In order to apply the two statistical fracture models to analyze the lifetime distribution of several long-term loading conditions given in the current work, it is first necessary to determine the values of the Weibull modulus $m$ and the subcritical crack growth exponent $n$. This can be done by evaluating the results of four-point small specimens subjected to rapid loading and sustained constant loads. Figure 1 shows a Weibull plot of the four-point flexure strength of fifteen small specimens. From the slope of the best-fit straight line obtained by linear regression analysis, the value of $m$ was determined to be 5.5 . Such a low value of $m$ indicates substantial scatter of the strength values. The mean strength value for the four-point small specimens was $254 \mathrm{MPa}$ with a standard deviation of $65 \mathrm{MPa}$. Fifteen specimens might be barely enough to determine the $m$ value as long as this group of specimens contain a representative number of strength-limiting defects. SEM analysis of the fracture surfaces found that the failure origins for specimens in both fast and time-dependent fracture testing were associated with similar strength-limiting defects. Therefore, the $m$-value obtained from these 15 specimens are acceptable for further use in the fatigue lifetime analysis. Similar situations were also reported in some of the previous

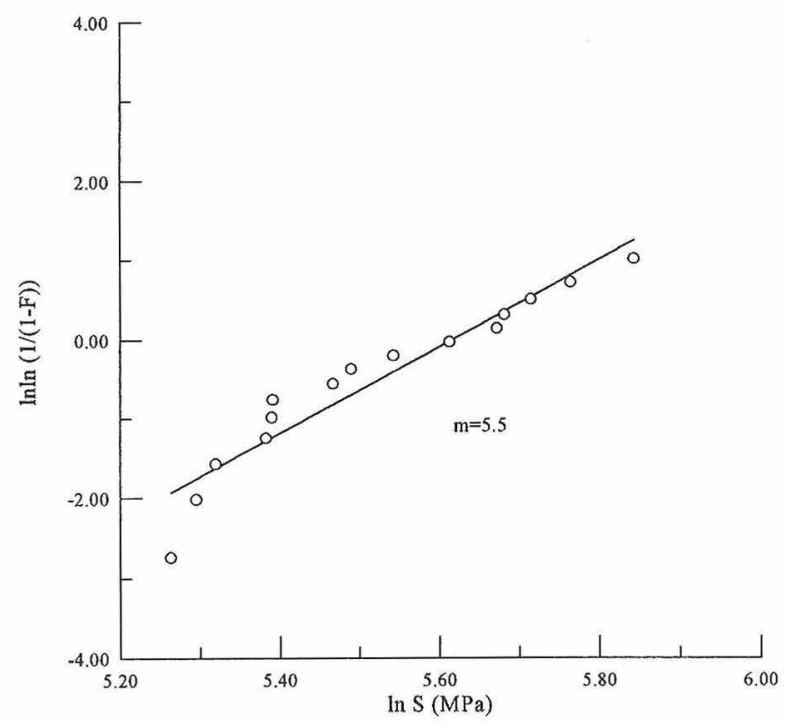

Fig. 1. Weibull plot of the flexural strength of an alumina in fourpoint bending. studies $^{9), 10), 12)}$ in which some $17-19$ specimens were used to determine the $m$-value for statistical analysis of fatigue lifetime without losing the accuracy. In addition, SEM observation also indicated that the fracture origins for specimens in both fast and delayed fracture testing were associated with surface flaws as well as volume defects. Surface flaws were usually open pores but in some cases no fracture origins were clearly defined which were often found originating on the surface. However, the volume defects might include a couple of types. Examples of the fracture origins at the volume defects observed in the flexure specimens are shown in Fig. 2. In this regard, it is appropriate to use the scaling relationships derived in Eqs. (4) and (5) of volume defects for further fatigue lifetime analysis based on a more general consideration. Therefore, the low $m$-value obtained might reflect the statistically averaged flaw characteristics of both surface and volume defects.

The stress-life data from static fatigue tests on fourpoint small specimens are analyzed to establish the reference fracture mechanics data such as $n$ and other parameters used in the lifetime prediction. The single-crack model is first adopted to analyze the stress-life data, then the multiple-crack model. A linear relationship between $\ln t_{\mathrm{sf}}$ and $\ln \sigma_{\mathrm{m}}$ can be observed in Eq. (10), given a failure probability $F$ and the associated fracture parameters $m, \sigma_{0}$, $A, n$ and $K_{\text {IC }}$. Without measuring these fracture parameters individually we can take another approach as follows. Equation (10) can be rearranged to give

$$
\ln \ln \frac{1}{1-F}=\frac{m}{n-2} \ln t_{\mathrm{sf}}+\frac{m n}{n-2} \ln \sigma_{\mathrm{m}}+\ln C_{\mathrm{s}}
$$

where

$$
\begin{aligned}
\ln C_{\mathrm{s}}= & -\ln \left\{\frac{4(m+1)^{2} \sigma_{0}^{m}}{(m+2) V}\right. \\
& \left.\times\left[\frac{2}{(n-2) A Y^{2} K_{\mathrm{IC}}^{n-2}}\right]^{m / n-2}\right\}
\end{aligned}
$$

Therefore, $\sigma_{0}, A$ and $K_{\text {IC }}$ need not be determined separately but can be evaluated as a combined parameter given by $\ln C_{\mathrm{s}}$ which is a constant term for a given material and specimen size.

Taking the lifetime distribution at each static stress level of 230,210, 190 and $170 \mathrm{MPa}$ and fitting each set of data according to the Weibull distribution as expressed in Eq. (20), we can determine the values for the coefficients $m /(n-2)$ and $m n /(n-2)$ by multiple linear regression (MLR) analysis. With the previously obtained $m$-value, $n$ is thus determined from the best-fit values of $m /(n-2)$ and $m n /(n-2)$.
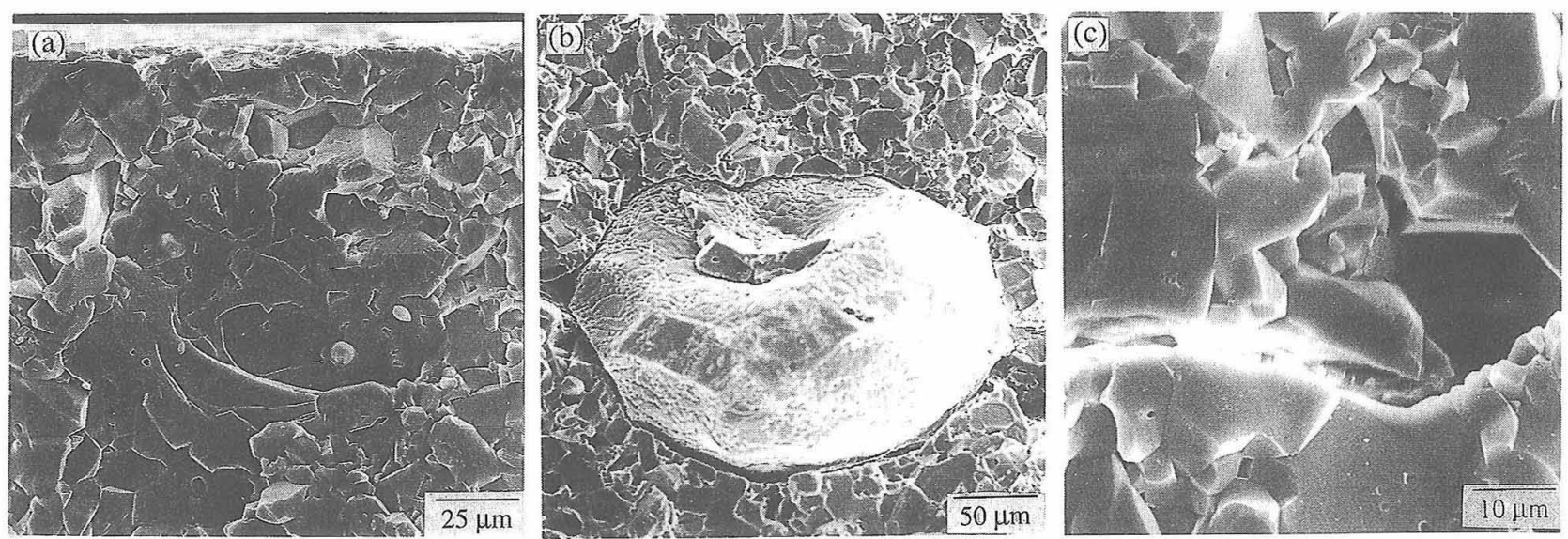

Fig. 2. Examples of volume defects observed as origins for fast and delayed fractures: (a) a cluster of large grains, (b) an agglomerate associated with porosity and (c) a pore. 
Since $m$ has been determined as 5.5, the crack growth exponent $n$ was accordingly determined as 36 from the MLR best-fit results for the 35 static fatigue data points of small specimens generated at four stress levels. This $n$-value is very close to those reported in direct crack growth tests. The typical values of $n$ for alumina generally found in direct crack growth tests ${ }^{18)-20)}$ are within the range of $15-40$. However, if Eq. (8) without statistics is used instead, the $n$ value determined from the simple $\ln \sigma_{\mathrm{m}}-\ln t_{\mathrm{sf}}$ plot is 120 which is much greater than those obtained for direct crack growth tests and will generate different, unreliable pictures of lifetime predictions for the other loading conditions discussed below. Similar observation was also reported in a previous study ${ }^{11)}$ in which the $n$-value was reduced to 25 from 140 with a consideration of statistics. It is noted that the value of $n$ here is determined from a total of 35 data points generated at four stress levels such that the statistical variability is much less than that determined based on a single or a couple of stress levels only. Therefore, the accuracy of determining the $n$-value as described above is acceptable and it is appropriate to use this $n$-value for further fatigue lifetime prediction.

By such an appropriate determination of $n=36$ together with the corresponding $C_{\mathrm{s}}$-value, the strength-probabilitylifetime relationship for the static data can be plotted in Fig. 3 in accordance with Eq. (10). Note that in Fig. 3 each data point represents a single specimen tested to failure except for those with labels indicating repeated data points. Left arrows designate specimens that broke on the loading ramp while right arrows designate runout tests. Apparent$1 y$, the statistical fracture approach of the single-crack model applied here describes the scatter of the stress-life data reasonably well, as most of the 35 data points are enclosed between the two lines which represent failure probabilities of $F=0.9$ and $F=0.1$, respectively. It is not surprisingly to find a very wide scatter band of fatigue lifetime for this alumina because of its low $m$-value. As mentioned previously, this low $m$-value represents a large scatter of the strength-limiting flaw characteristics (such as types and sizes). Therefore, for ceramics with low values in Weibull modulus $m$ and a large scatter in strength, statis-

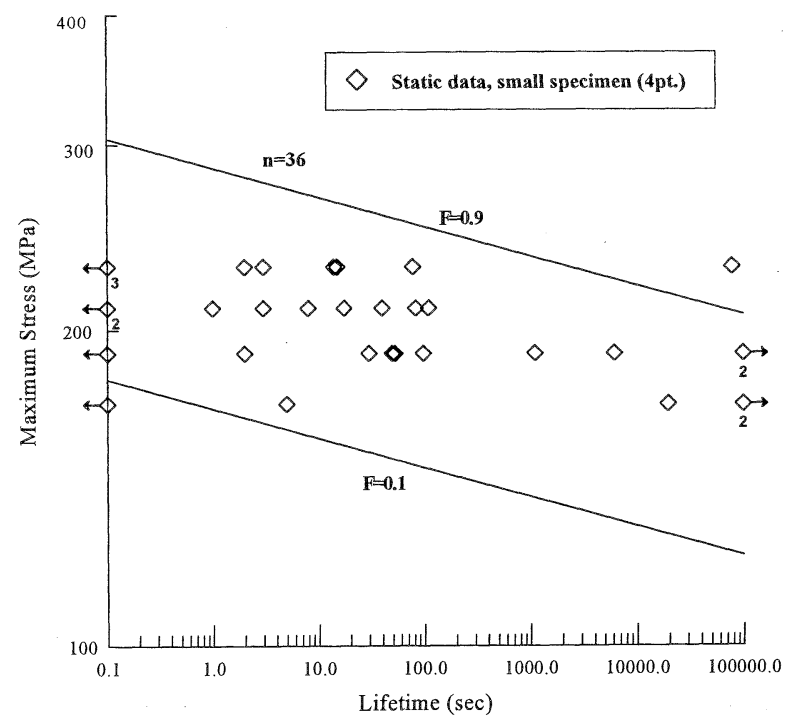

Fig. 3. Relationship between applied static stress and lifetime for small specimens under four-point flexure according to the statistical single- and multiple-crack models (Arrows at right designate runout tests; at left breakages during ramp loading to maximum applied stress. Number labels represent repeated data points. Same denotation in the subsequent figures). tics must be considered in describing the stress-life relationship of delayed fracture for more reliable lifetime prediction.

Similarly, for the multiple-crack model, the following equations can be obtained from Eq. (16)

$$
\ln \ln \frac{1}{1-F}=\frac{m}{n-2} \ln t_{\mathrm{sf}}+\frac{m n}{n-2} \ln \sigma_{\mathrm{m}}+\ln C_{\mathrm{m}}
$$

where

$$
\begin{aligned}
\ln C_{\mathrm{m}}= & -\ln \left\{\frac{4\left(m_{*}+1\right)^{2} \sigma_{\mathrm{o}}^{m}}{\left(m_{*}+2\right) V}\right. \\
& \left.\times\left[\frac{2}{(n-2) A Y^{2} K_{\mathrm{IC}}^{n-2}}\right]^{m / n-2}\right\}
\end{aligned}
$$

Note that Eq. (22) is analogous to Eq. (20) except for the difference in the contents of the constant terms $C_{\mathrm{s}}$ and $C_{\mathrm{m}}$. However, fitting the static fatigue data of small specimens according to the relationships of Eqs. (22) and (20) by MLR analysis generates the same values for $m /(n-2)$ and $m n /(n-2)$, and also identical values for the constant terms, $C_{\mathrm{s}}$ and $C_{\mathrm{m}}$. In this regard, both single-crack and multiple-crack models would provide the same fitted stress-life relationship for the data from small specimens under fourpoint flexure in the static fatigue tests. Apparently, both models performed well in consolidating the statistical concepts to present the stress-life relationship in delayed fracture for the given testing conditions. It is realized that the accuracy of lifetime prediction, whether based on the single- or multiple-crack model, depends on the accuracy of determining the value of the subcritical crack growth exponent $n$. Again, from the results shown in Fig. 3 , the accuracy of estimation for $n$-value as described above in the current study is acceptable.

\subsection{Evaluating the effect of cyclic loading}

It is necessary to examine whether selected alumina exhibits any true cyclic fatigue effect so as to provide the justification basis for applying the two statistical fracture models in cyclic lifetime prediction. The lifetime prediction in brittle materials subjected to cyclic stresses can be achieved from the static lifetime data provided that cyclic fatigue-induced damage can be neglected and the subcritical crack growth effect becomes significant. From Eqs. (10) and (11) the single-crack theory gives

$$
r_{1}=\frac{t_{\mathrm{cf}}}{t_{\mathrm{sf}}}=\tau\left\{\int_{0}^{\tau}[f(t)]^{n} \mathrm{~d} t\right\}^{-1}
$$

Here $r_{1}$ is the lifetime ratio of cyclic loading to static loading under the same maximum applied stress $\sigma_{\mathrm{m}}$ given a failure probability. A similar lifetime ratio expressed in Eq. (24) can also be obtained for the multiple-crack model using Eqs. (16) and (17). In this regard, both models make the same prediction of cyclic lifetime from static lifetime if there is no existence of a true cyclic effect. It is noted that $r_{1}$ is independent of frequency due to the assumption that cyclic fatigue is an integrated effect of static fatigue damage through time accumulation.

For the given cyclic fatigue testing conditions and the previously obtained $n$-value, Eq. (24) gives a value of 10 for $r_{1}$. Using this $r_{1}$-value and the best-fit static stress-life relationship obtained in the previous section, cyclic lifetimes for small specimens under four-point flexure are readily estimated. The predicted cyclic lifetimes for four-point small specimens are then plotted in Fig. 4 to compare with the experimental results. The general agreement between the predicted values and the experimental data is not unreasonable, as the majority of the 20 cyclic data points generated at four stress levels are predictable and located within the region bound by the two predicted lines, $F=0.9$ 


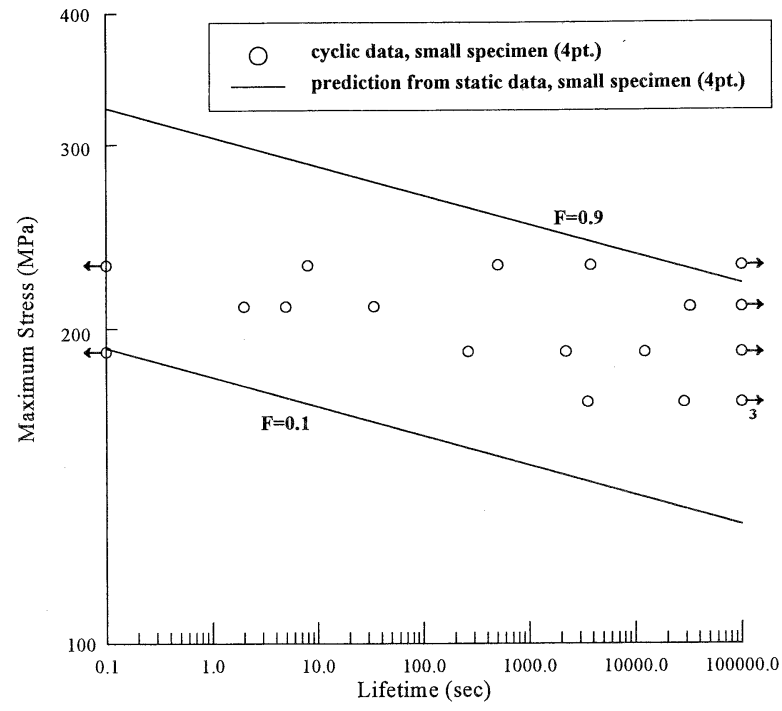

Fig. 4. Comparison of cyclic lifetime data for small specimens under four-point flexure with predictions from static lifetime data of small specimens under four-point flexure in accordance with the single- and multiple-crack models.

and $F=0.1$. This implies that cyclic loading might not cause more detrimental damage than static loading for the given alumina and testing conditions with statistical consideration. In other words, any subsidiary cyclic damage mechanism might play an insignificant role in the degradation process. Therefore, the delayed fracture behavior of this alumina under both static and cyclic loading is predominantly time-dependent. This reasonable agreement between the theoretically predicted lifetimes and experimental results suggests that fracture statistics together with the powerlaw crack growth relationship used in both the single- and multiple-crack models might be applied to selected alumina for cyclic lifetime prediction. The applicability of these two models to predict the size effects on the lifetime distribution of delayed fracture is discussed below.

4.3 Evaluation of size effect between large and small specimens under four-point flexure

Estimation of size effects on the static and cyclic fatigue lifetimes in brittle materials under four-point flexure can be obtained through the strength-probability-lifetime relationships given in Eqs. (10), (11), (16) and (17). From these equations both the single- and multiple-crack models give

$$
r_{2}=\frac{t_{\text {sf(large })}}{t_{\text {sf }(\text { small })}}=\frac{t_{\text {cf(large })}}{t_{\mathrm{cf}(\text { small })}}=\left[\frac{V_{\text {(small })}}{V_{\text {(large })}}\right]^{n-2 / m}
$$

Here $r_{2}$ is the lifetime ratio of large specimen to small specimen under the same maximum applied static or cyclic stress $\sigma_{\mathrm{m}}$ given a probability. Therefore, both models on four-point flexure estimate the same effects of specimen size on the fatigue lifetime in ceramics under long-term static or cyclic loading. Equation (25) predicts a decrease in lifetime with increasing specimen size by a factor of $r_{2}$ in each loading mode.

For the previously determined values of $n=36$ and $m=5.5$ together with the given volume ratio of the large specimen over the small specimen (this value is 9), Eq. (25) gives a value of $1.26 \times 10^{-6}$ for $r_{2}$. This means that both models predict a much smaller fatigue lifetime for the large specimen by a magnitude of about six orders as compared to the small specimen under the same maximum applied stress and failure probability. Static lifetimes for large specimens are readily estimated by using this $r_{2}$-value (Eq. (25)) and the best-fit static stress-life relationship (Eq.
(10)) for small specimens obtained previously. The predictions for large specimens under four-point static loads are then plotted in Fig. 5 to compare with the results from experiments. Similar comparison of cyclic lifetimes for large specimens under four-point flexure can be made by the same procedures, as shown in Fig. 6. In Fig. 6, the prediction is fairly good as the experimental data points are mostly encompassed by the two predicted lines, $F=0.1$ and $F=0.9$. However, in Fig. 5 the static fatigue lifetimes for higher applied stress levels are somewhat underestimated. This deviation from the prediction at high applied stresses is due to the insignificant effect of subcritical crack growth for short fatigue lifetime. Nevertheless, the general description of the scatter of the large specimen data in both fourpoint static and cyclic fatigue by the two statistical fracture approaches used here is acceptable. This suggests that both the single-and multiple-crack models are applicable to

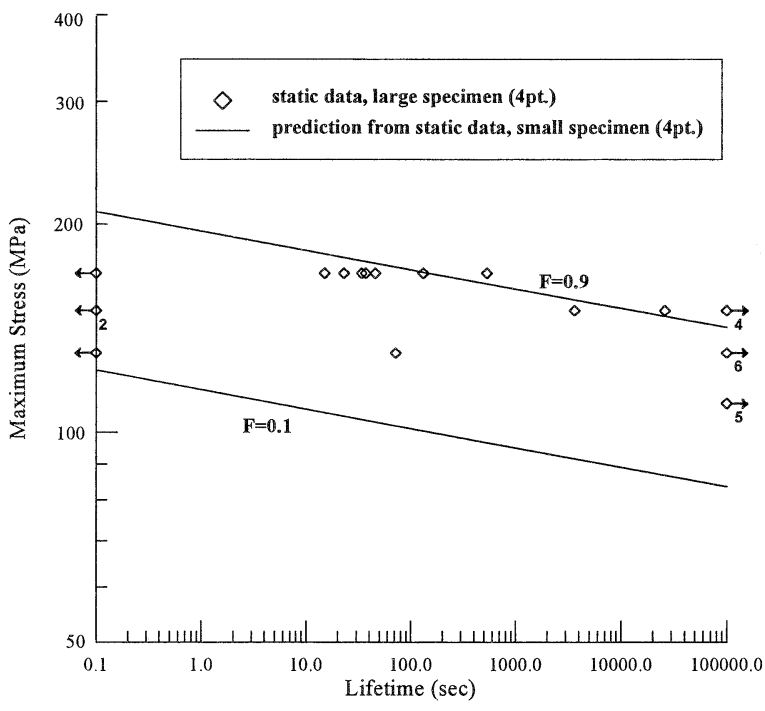

Fig. 5. Comparison of static lifetime data for large specimens under four-point flexure with predictions from static lifetime data of small specimens under four-point flexure in accordance with the single- and multiple-crack models.

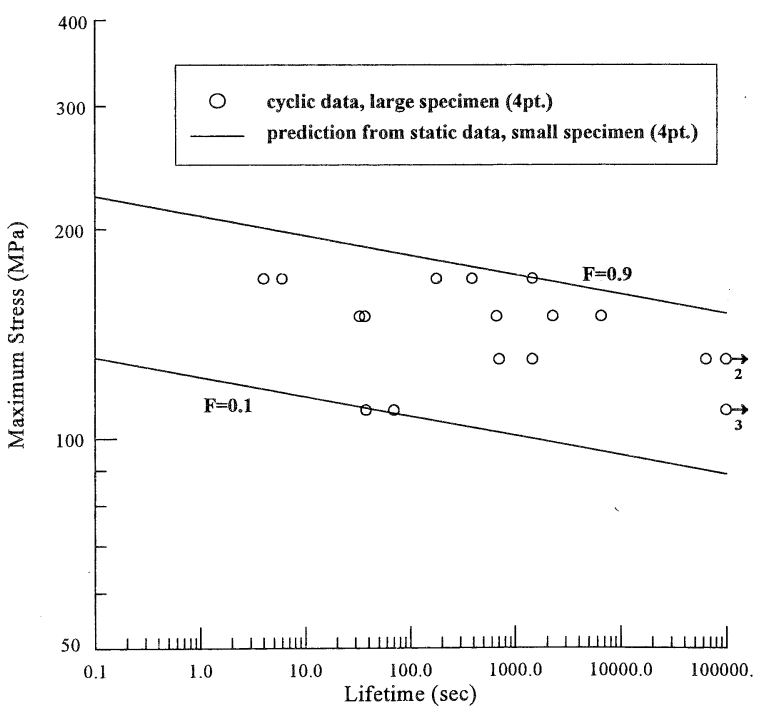

Fig. 6. Comparison of cyclic lifetime data for large specimens under four-point flexure with predictions from static lifetime data of small specimens under four-point flexure in accordance with the single- and multiple-crack models. 
evaluate the size effects on the time-dependent fracture of selected alumina. It also implies that time-dependent fracture in both small and large specimens under four-point flexure might be controlled by the same flaw statistics. It is again emphasized that both the single- and multiple-crack models predict the same size effects in the present case, since the intrinsic differences in these two theories are lost by MLR fitting of the stress-life data in determining the values of the constant terms $C_{\mathrm{s}}$ and $C_{\mathrm{m}}$ in Eqs. (20) and (22), respectively.

4.4 Evaluation of size effect between four- and threepoint flexure modes

Another size effect on ceramic strength is related to the difference in stress distribution. The application of the two statistical fracture models to predict this type of size effect on the fatigue lifetime for various stress distributions (such as four-point flexure vs. three-point flexure) is assessed by analyzing the static and cyclic fatigue data from three-point flexure tests on large specimens. From Eqis. (10) and (12) for static fatigue and Eqs. (11) and (13) for cyclic fatigue, the single-crack theory gives

$$
r_{3(\mathrm{sc})}=\frac{t_{\mathrm{sf}(3 \mathrm{pt})}}{t_{\mathrm{sf}(4 \mathrm{pt})}}=\frac{t_{\mathrm{cf}(3 \mathrm{pt})}}{t_{\mathrm{cf}(4 \mathrm{pt})}}=\left(\frac{m+2}{2}\right)^{n-2 / m}
$$

Here $r_{3}$ is the fatigue lifetime ratio of three-point flexure to four-point flexure under the same maximum applied static or cyclic stress $\sigma_{\mathrm{m}}$ given a specimen size and failure probability. However, the multiple-crack theory will give different lifetime ratio between three- and four-point flexures because of the different assumptions on the flaw size distribution and growth characteristics during fatigue testing. From Eqs. (16) and (18) for static fatigue and Eqs. (17) and (19) for cyclic fatigue, the fatigue lifetime ratio based on the multiple-crack theory is given by

$$
r_{3(\mathrm{mc})}=\frac{t_{\mathrm{sf}(3 \mathrm{pt})}}{t_{\mathrm{sf}(4 \mathrm{pt})}}=\frac{t_{\mathrm{cf}(3 \mathrm{pt})}}{t_{\mathrm{cf}(4 \mathrm{pt})}}=\left(\frac{m_{*}+2}{2}\right)^{n-2 / m}
$$

The difference between Eqs. (26) and (27) is the terms of $m$ and $m_{*}$ as a result of the different assumptions between the two models. This is so far the only difference in lifetime prediction from these two models for the given testing conditions.

For materials obeying the single- or multiple-crack model, the size effect from various stress distributions in three- and four-point flexures can be obtained explicitly from Eq. (26) or (27). Both models predict a longer lifetime for three-point flexure by a factor $r_{3}$ over four-point flexure due to the less effective stressed volume in threepoint flexure. Substituting the values of $m=5.5$ and $n=36$ into Eqs. (26) and (27) gives a value of 3536 for $r_{3(\mathrm{sc})}$ and 4578 for $r_{3(\mathrm{mc})}$. These two values together with the previously obtained values of $r_{1}$ and $r_{2}$ will be applied to estimate the static and cyclic fatigue lifetime distributions for threepoint large specimens from the static fatigue data obtained for four-point small specimens. The prediction from singlecrack theory is performed and discussed first. The predicted static fatigue lifetime for three-point large specimens can be obtained by combining Eqs. (25) and (26) with the best-fit static stress-life relationship (in accordance with Eq. (10)) obtained for four-point small specimens. The predicted results are compared with the experimental data in Fig. 7. Prediction for cyclic fatigue lifetime can be obtained in the same way through Eqs. (10), (24), (25) and (26) and is plotted in Fig. 8 to compare with the experimental data. Similar procedures can be taken to make the predictions on static and cyclic fatigue lifetime distributions for large specimens in three-point flexure from the multiplecrack theory by using Eqs. (10), (24), (25) and (27). These predictions are also shown in Figs. 7 and 8 for static

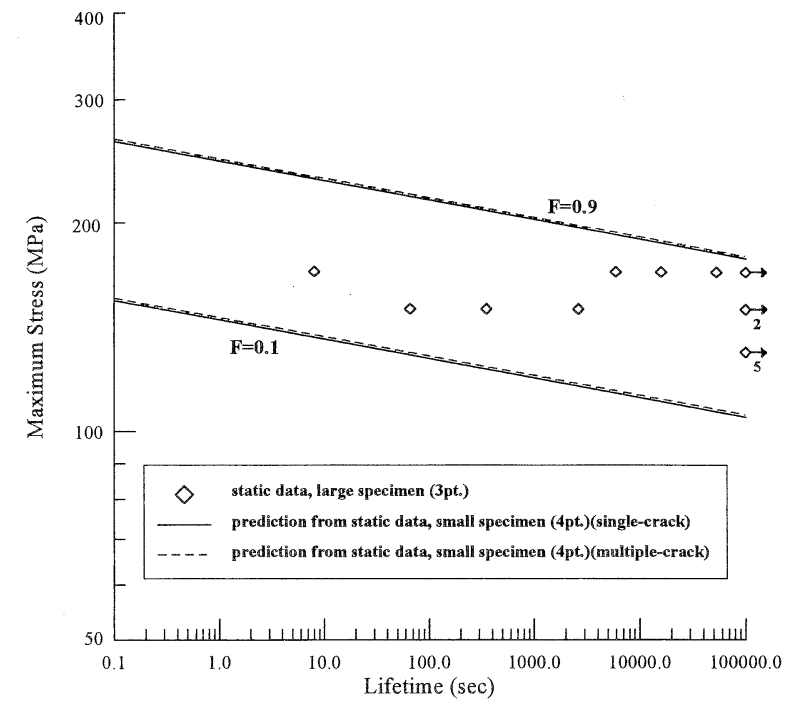

Fig. 7. Comparison of static lifetime data for large specimens under three-point flexure with predictions from static lifetime data of small specimens under four-point flexure in accordance with the single- and multiple-crack models.

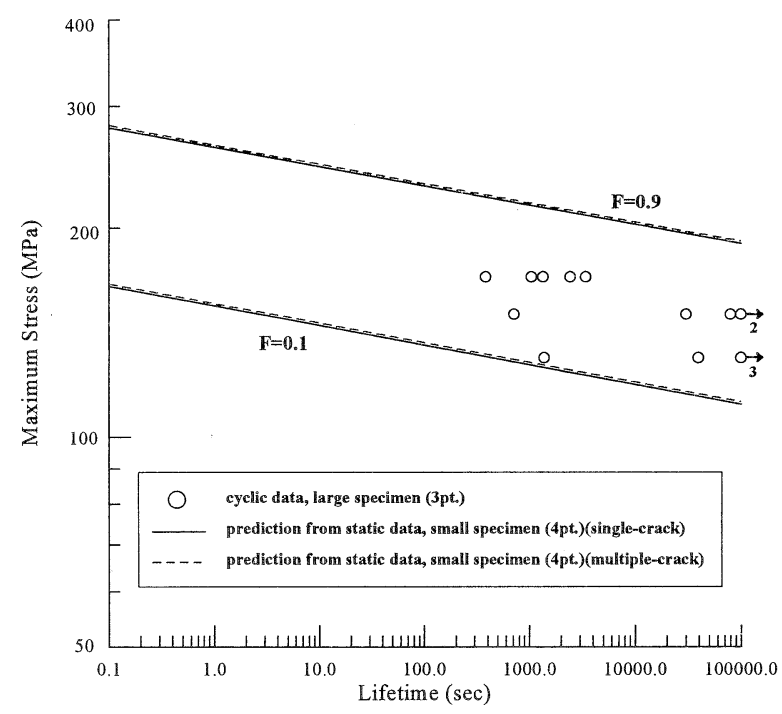

Fig. 8. Comparison of cyclic lifetime data for large specimens under three-point flexure with predictions from static lifetime data of small specimens under four-point flexure in accordance with the single- and multiple-crack models.

and cyclic fatigue, respectively, to compare with the experimental results and those made by the single-crack model.

As shown in Figs. 7 and 8, the experimental data points are mostly bound by the predicted lines, $F=0.1$ and $F=0.9$, made by each model. Accordingly, the size effect on delayed fracture, as derived from the static and cyclic data of large specimens under three-point flexure, is predictable through both the single- and multiple-crack models. This suggests that fracture of selected alumina in four-and three-point flexures might be caused by subcritical crack growth of flaws from the same statistical population. However, no significant difference in the predictions between these two models can be detected in this case because the difference in the values of $r_{3(\mathrm{sc})}$ and $r_{3(\mathrm{mc})}$ is very small in logarithmic scale (log 3536 vs. $\log 4578$ ). The general agreement between the statistical theory predictions and the experimentally measured lifetime data for the 
three-point large specimens under static and cyclic fatigue loading is acceptable. This encourages the validity of the two statistical fracture models in assessing size and stress distribution effects on the delayed fracture of selected alumina.

In summary, the fatigue lifetime data are greatly scattered for all of the given testing conditions, and it is difficult to estimate the fatigue behavior of the alumina without conducting a statistical analysis. Both the single- and multiplecrack models are not unreasonable in describing the strength-probability-lifetime relationships under time-dependent loads and in assessing the static and cyclic lifetime for the given alumina with various specimen sizes and stress distributions. However, we cannot tell which model is superior in the present study as only the simple stresslife test method is applied in the present work. If more data are available from direct measurements of the fracture parameters such as $\sigma_{0}, A, n$ and $K_{\text {IC }}$ for the selected alumina, the distinction between these two models may be determined. The applicability of these two models for making lifetime prediction on delayed fracture of ceramic components subjected to multi-axial stress states needs to be critically assessed in the future.

\section{Conclusions}

(1) Lifetime distributions of an alumina under threeand four-point flexure tests on different specimen sizes subjected to static and cyclic fatigue loads were reasonably described by two statistical time-dependent fracture models.

(2) The cyclic fatigue failure was time-dependent rather than cycle-dependent for selected alumina as the scatter of the cyclic fatigue lifetime was predictable by two statistical time-dependent fracture models from data obtained under sustained constant stresses without enhanced cyclic fatigue-induced effect considered.

(3) The effects of specimen size and stress distribution on the static and cyclic fatigue lifetime distribution for the given alumina can be reasonably estimated by both the single- and multiple-crack models.

(4) Both the single- and multiple-crack models are acceptable in describing the lifetime dispersion for all of the given testing conditions in the present study. Therefore, the different assumptions of flaw growth characteristics in both models might be valid for the given alumina and testing conditions.

\section{References}

1) D. G. S. Davies, Proc. Brit. Ceram. Soc., 22, 429-52 (1973).

2) G. K. Bansal and W. H. Duckworth, "Fracture Mechanics of Ceramics, Vol. 3," Ed. by R. C. Bradt, D. P. H. Hasselman and F. F. Lange, Plenum Press, New York (1978) pp. 189204.

3) D. K. Shetty, A. R. Rosenfield and W. H. Duckworth, "Methods for Assessing the Structural Reliability of Brittle Materials, ASTM STP 844," Ed. by S. W. Freiman and C. M. Hudson, American Society for Testing and Materials, Philadelphia (1984) pp. 57-80.

4) R. W. Davidge, J. R. McLaren and G. Tappin, J. Mater. Sci., 8, 1699-705 (1973).

5) P. F. Nicholls and A. Paluszny, "Ceramics for High Performance Applications," Ed. by J. J. Burke, A. E. Gorum and R. N. Katz, Book-Hill Publishing Co., Chestnut Hill, MA (1974) pp. $63-78$.

6) A. G. Evans and S. M. Wiederhorn, Int. J. Fracture, 10, 37992 (1974).

7) K. Jakus, D. C. Coyne and J. E. Ritter, Jr., J. Mater. Sci., 13, 2071-80 (1978).

8) X.-Z. Hu, Y.-W. Mai and B. Cotterell, Philos. Mag. A, 58, 299-324 (1988)

9) X.-Z. Hu, Y.-W. Mai and B. Cotterell, J. Mater. Sci., 24, 3118-22 (1989).

10) Y. Matsuo, T. Oida, K. Jinbo, K. Yasuda and S. Kimura, J. Ceram. Soc. Japan, 97, 136-42 (1989).

11) T. Ogasawara, Y. Akimune and K. Yoneda, J. Ceram. Soc. Japan, 99, 489-94 (1991).

12) T. Ogasawara, N. Hirosaki and Y. Akimune, J. Am. Ceram. Soc., 77, 514-18 (1994).

13) W. Weibull, J. Applied Mech., 18, 293-97 (1951).

14) R. A. Hunt and L. N. McCartney, Int. J. Fracture, 15, 365-75 (1979).

15) S. M. Wiederhorn, "Fracture Mechanics of Ceramics, Vol. 2," Ed. by R. C. Bradt, D. P. H. Hasselman and F. F. Lange, Plenum Press, New York (1974) pp. 623-46.

16) A. G. Evans and E. R. Fuller, Metall. Trans., 5, 27-33 (1974)

17) C.-K. Lin, C.-T. Chiu and W.-S. Chen, J. Chinese Soc. Mech. Eng., 17, 133-43 (1996).

18) K. E. Aeberli and R. D. Rawlings, J. Mater. Sci. Lett., 2, 21520 (1983).

19) M. J. Reece, F. Guiu and M. F. R. Sammur, J. Am. Ceram. Soc., 72, 348-52 (1989).

20) R. H. Dauskardt, J. Engr. Mater. Tech., 115, 244-51 (1993). 